\title{
Assessing the kinetic model of hydro-distillation and chemical composition of Aquilaria malaccensis leaves essential oil
}

\begin{abstract}
This study aimed to model the kinetic of hydro-distillation of Aquilaria malaccensis leaves oil in order to understand and optimize the extraction process. In addition, this study, for the first time, aimed to identify the chemical compositions of the A. malaccensis leave-oil. By assessing both first-order kinetic model and the model of simultaneous washing and diffusion, the result indicated that the model of simultaneous washing and diffusion better describes the hydro-distillation mechanism of the essential oil from A. malaccensis leaves. The optimum time, solid to liquid ratio, and the heating power for extracting the highest amount of essential oil were found to be around $3 \mathrm{~h}, 1: 10(\mathrm{~g} \cdot \mathrm{ml}-1)$, and $300 \mathrm{~W}$ respectively. Yellow essential oil with a strong smell and a yield of $0.05 \mathrm{v} / \mathrm{w}$ was extracted by hydrodistillation Clevenger apparatus. Chemical compounds of the essential oil were analyzed using gas chromatography-mass spectroscopy (GC/MS), which resulted in identification of 42 compounds that constitute $93 \%$ of essential oil. Among the identified components, Pentadecanal (32.082\%), 9-Octadecenal, (Z) (15.894\%), and Tetradecanal (6.927\%) were the major compounds. Considering the fact that all the identified major components possess pesticidal properties, A. malaccensis leaves can be regarded as a promising natural source for producing pesticides.
\end{abstract}

Keyword: Aquilaria malaccensis; Essential oil; Hydro-distillation; Optimization; Kinetic modeling 